\title{
Linx
}

Revue des linguistes de l'université Paris X Nanterre

56 | 2007

Linguistique des genres

\section{Contribution à une linguistique néo-saussurienne des genres de la parole (1) : une grammaire du morphème on}

\section{Simon Bouquet}

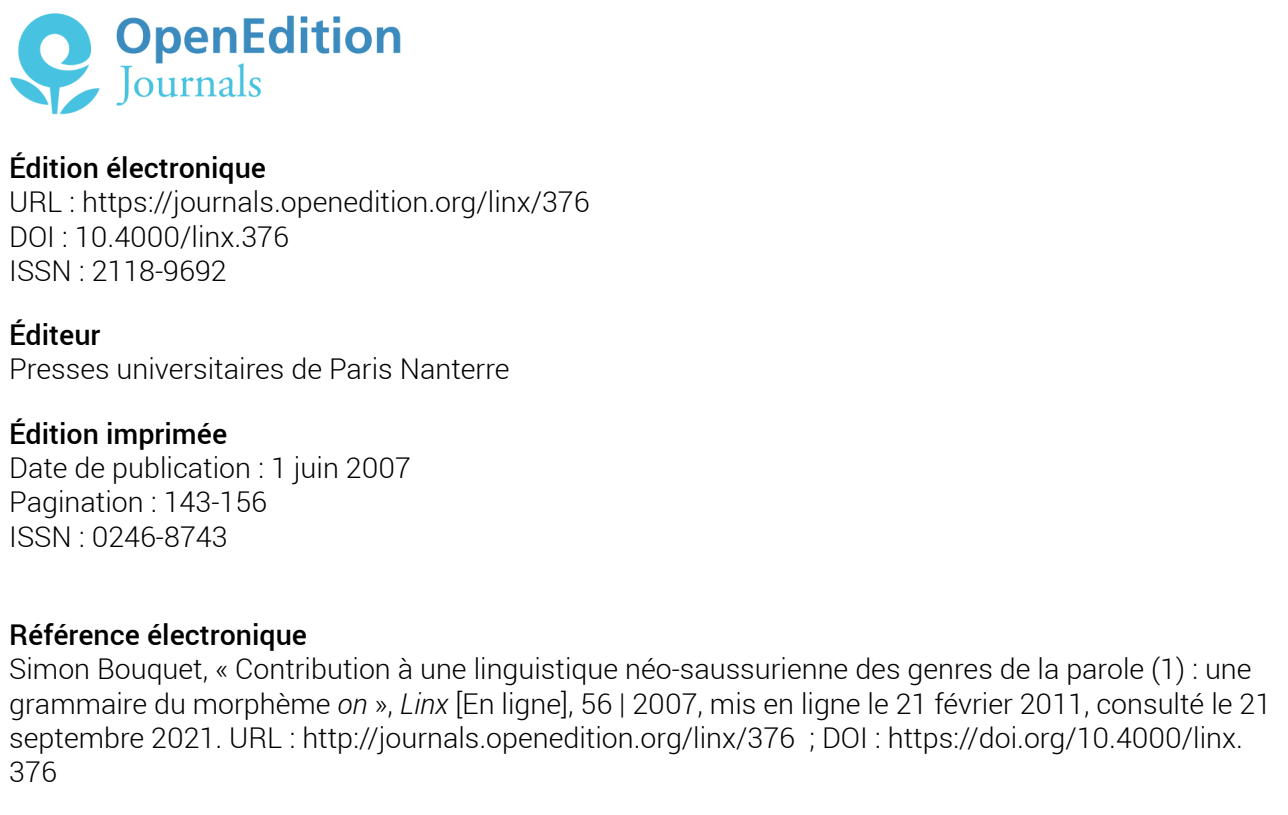

Département de Sciences du langage, Université Paris Ouest 


\title{
Contribution \\ à une linguistique néo-saussurienne des genres de la parole (1) : une grammaire du morphème on
}

\author{
Simon Bouquet, Université Paris X-Nanterre - CNRS MoDyCo
}

\begin{abstract}
Le mécanisme de la langue - prise partout À UN MOMENT DONNÉ, ce qui est la seule manière d'en étudier le mécanisme - sera un jour, nous en sommes persuadé, réduit à des formules relativement simples. (...) Cette distinction, quand on arrive au détail, est tellement délicate qu'elle absorbe à elle seule une attention, même très soutenue, qu'elle sera probablement traitée de distinction subtile dans mille cas, prévus ou imprévus.

Ferdinand de Saussure, De l'essence double du langage ${ }^{2}$
\end{abstract}

La diversité successive des combinaisons linguistiques (dites états de langue) (...) ou ne comporte rien, ou comporte une description et une appréciation mathématique, mais elle ne comporte pas de dissertations flottantes portant sur l'extérieur (...)

Ferdinand de Saussure, Notes pour un article sur Whitney ${ }^{3}$

Cet article forme un triptyque avec ceux de Denise Malrieu et de Créola Thénault. Notre propos commun est d'illustrer une linguistique unissant inséparablement analyse de la langue et analyse de la parole, fondée sur le programme des

1 Cet article est le fruit d'une collaboration avec Denise Malrieu, que je tiens à remercier chaleureusement. Merci également à Annie Delaveau et à Danielle Leeman pour leurs commentaires réconfortants et leurs suggestions.

${ }^{2}$ Ecrits de linguistique générale, Paris, Gallimard, 2002, p. 43.

${ }^{3}$ Ecrits de linguistique générale, p. 206-207. 
textes saussuriens originaux redécouverts aujourd'hui. Cette démarche, selon nous, est propre à donner un contenu formel au projet bakhtinien d'une linguistique des genres. (Cf. introduction supra).

Nous nous attacherons, dans cette perspective, à une étude du morphème clitique on. Ma contribution, relevant d'une linguistique de la langue, propose une grammaire sémiotique des valeurs de on dans le système du français. L'article de Denise Malrieu analyse, dans un corpus de textes de Marguerite Duras et de Stendhal, l'actualisation des valeurs de on mises en évidence par cette grammaire. L'article de Créola Thénault examine, dans un sous-ensemble du corpus de Denise Malrieu, la traduction du on français en roumain.

\section{Remarques liminaires}

\subsection{Langue et parole}

Se cantonnant à une grammaire de langue, le présent article décrit des valeurs des entités virtuelles d'un système synchronique - qui peuvent et doivent, selon le programme épistémologique de Saussure, être représentées sous la forme d'une algèbre. Il s'ensuit que l'essence de notre grammaire réside dans la désignation de ces valeurs par des numéros, considérés comme une littéralisation ${ }^{4}$. La base binaire de notre numérotation rend compte du caractère strictement différentiel des valeurs en question, toute valeur «1» de nos arborescences renvoyant à la négation réciproque d'une valeur « 2 » de même niveau ${ }^{5}$.

Les valeurs de langue ainsi définies sont illustrées par des exemples, sans lesquels notre grammaire resterait peu lisible. Ces « exemples de grammaire », comme le veut le genre particulier auquel ils appartiennent, supposent de la part du lecteur une construction, plus ou moins consciente, de situations de parole. Conformément à la tradition grammaticale, ces situations de parole resteront passablement implicites à ce palier de description.

Il n'en ira pas de même dans les contributions de Denise Malrieu et de Créola Thénault, qui mettront notre grammaire de langue à l'épreuve d'une grammaire de « valeurs

\footnotetext{
${ }^{4}$ Sur la notion de «littéralisation » et les présupposés « galliléens » de notre perspective épistémologique, cf. J.-C. Milner, Introduction à une science du langage, Paris, Editions du Seuil, 1989 (première partie), et S. Bouquet, Introduction à la lecture de Saussure, op.cit. (notamment le préambule). - Ainsi Saussure définissait-il l'épistémologie sous-tendant la grammaire comparée, au regard de la notation d'un élément phonologique d'une langue: «il pourra, une fois pour toutes, être catalogué par un numéro représentant une valeur quelconque, pourvu qu'elle ne se confonde pas avec celle des autres numéros" (Cours de linguistique générale, édition critique par Rudolf Engler, Wiesbaden, Otto Harrassowitz, 1968, p. 496 - notes du cours de 1907).

${ }^{5}$ Pour préserver la lisibilité de notre grammaire, la présentation des valeurs dans cet article est simplifiée. On se rappellera que la formule algébrique des signifiés tient, outre à la négation réciproque notée par chiffres 1 et 2 , au niveau occupé par ces chiffres dans l'arborescence unique de on. Ainsi, par exemple, la formule complète de on $=$ «tu » (masculin) - sommant les arborescences I et IIa et notant le niveau de l'arborescence en indice - serait :$$
{ }_{1} 1_{2} 1_{3} 1_{4} 1{ }_{5} 2{ }_{6} 1{ }_{7} 1
$$

correspondant à : +SEM/+IDX/+EXT/+ITL/-LOC/-EMP/+MASC
} 
de parole » fondée sur le même principe d'oppositivité (on appellera par commodité ces valeurs genres, mais ce vocable ne devra pas être référé à l'acception, passablement incertaine, qui est la sienne en linguistique aujourd'hui : il désigne pour nous, sans préjugé de substance, un simple « signifié suprasegmental » - un signifié s'étendant à la totalité d'une séquence de parole - noté en l'occurrence par un trait oppositif). Cette approche permettra d'établir une corrélation entre ce trait différentiel de genre et une valeur différentielle de on établie par notre grammaire de langue. Dans la perspective «galiléenne » où nous nous situons, cette corrélation s'entendra comme l'écriture formelle d'une "loi », appliquée aux valeurs différentielles de langue et de parole littéralisées par nos grammaires, et satisfaisant au critère de réfutabilité dans la mesure où cette loi s'oppose, par définition, à une autre loi (autrement dit : à une corrélation entre un autre trait de genre et une autre valeur différentielle de on) décrivant le sens différent d'une séquence de parole virtuelle, homonyme ( $f$. notre introduction supra).

\subsection{Sémiotique de la langue}

Dans la présente grammaire, les descripteurs des traits de signifié explicitant les valeurs différentielles ([+/-IDX], [+/-LOC], [+/-SING], etc.), quand bien même leur désignation fait-elle appel à des concepts grammaticaux largement partagés (« indexicalité », « locuteur », «singulier », etc.), ne doivent pas être considérés comme préexistants aux valeurs qu'il désignent, mais au contraire comme déterminés par la synchronie de la langue que cette grammaire fait apparaitre. De fait, si l'on accepte l'ontologie négative qui sous-tend la notion saussurienne de "valeur pure », c'est l'existence implicite d'un grammaire differentielle dans la compétence des sujets parlants - attestable par le fait qu'elle peut faire l'objet d'une écriture algébrique explicite - qui est à l'origine desdits concepts grammaticaux $x^{6}$.

En outre, nous fondant sur une conception sémiotique du système de la langue, nous adoptons comme première subdivision de cette grammaire - et de toute grammaire de langue - la trichotomie icône/indice/symbole proposée par Peirce pour définir le lien signe/sens. Dans la présente grammaire, l'iconicité, ne concernant que la position syntaxique commune à toutes les occurrences possibles de on, n'a pas de caractère différentiel. Notre subdivision initiale se limite donc à la subdivision du trait [-ICO] (iconicité) dans les traits [+IDX] (indexicalité) et [-IDX] (symbolisme).

\subsection{Le concept d' "empathie"}

Si nos traits descriptifs se fondent essentiellement sur des notions grammaticales et sémiotiques largement partagées, nous y introduisons toutefois un concept moins courant, celui d'« empathie ». Comme on le verra, l'opposition des traits [+EMP] (empathie) et [-EMP] subdivise certaines valeurs de nos arborescences : celles de l'amplification des personnes du dialogue (arborescences II.b. et II.c), celles de l'anaphore associative (arborescence III.) et celles de la valeur nominale, dite ici symbolique (arborescence IV.).

\footnotetext{
${ }^{6}$ L'originalité foncière de la pensée de Saussure tient à une thèse ontologique qu'on peut formuler ainsi : les valeurs de langue sont préalables à leurs « référents » et construisent ceux-ci comme tels. Sur cette thèse ontologique, se fonde son programme épistémologique : l’adoption de ce point de vue radical pour construire et décrire l'objet « langue ».
} 
Le concept grammatical d'« empathie » a acquis - notamment avec les travaux de Susumu Kuno ${ }^{7}$ et, plus récemment, avec ceux de Robert Forest ${ }^{8}-$ ses lettres de noblesse en linguistique. Nous l'entendons toutefois dans une acception plus générale que ces auteurs : ce terme réfère, dans nos arborescences, à la valeur spécifique d'un morphème susceptible de régir la construction d'une "sphère » (ou classe) d'association autour d'un "centre » (s'agissant d'une construction de signifié, le « centre », tout comme les entités associées de la «sphère », seront des valeurs). Ainsi, par exemple, l'amplification du trait interlocutoire [+LOC] dans le nous ou le on, tout comme l'amplification du trait interlocutoire [-LOC] dans le vous ou le $o n^{9}$, sont-elles traitées ici comme un cas particulier d'empathie linguistique, dans lequel le trait support de l'amplification désigne le centre d'empathie (respectivement les valeurs «standard» de je et de $t u$ ), et les valeurs de nous, vous, on ressortissent à une construction de sphère d'empathie autour de ce centre. Si l'on parle ici de construction, c'est pour rendre compte de ce que la sphère d'empathie, virtuelle, n'est pas fixée en elle-même par la valeur [+EMP] du morphème : cette valeur note simplement qu'une telle construction de sphère peut (et doit) avoir lieu. Si les constructions de sphères empathiques dont cette valeur note la possibilité sont en nombre indéfini, certaines n'en relèvent pas moins de régularités (ainsi qu'en témoigne, notamment, l'amplification du locuteur ou de l'allocutaire décrite dans nos arborescences II.b. et II.c.).

\section{1..4. Le morphème on}

L'unité de langue dont on présente ici une grammaire sémiotique ne sera spécifiée, hormis par sa forme phonologique, que de manière minimale. En effet, les préoccupations substantialistes qui sont habituellement celles des grammairiens - par exemple, leurs spéculations sur une valeur de on transversale à la diversité de ses emplois, ou encore sur sa nature grammaticale (pronom personnel ou pronom indéfini) - ne sont pas de mise dans la perspective strictement différentielle qui est la nôtre. Aussi s'en tiendra-t-on, quant à la délimitation de l'objet d'analyse - outre sa forme phonologique -, à une simple caractérisation syntaxique : le morphème on est un clitique verbal apparaissant en position argumentale sujet. De ce fait, on entretient des relations structurales avec d'autres morphèmes répondant à la même définition (notamment : je, tu, nous, vous, il, elle, ils, elles).

Le morphème on, s'il porte toujours le trait [+humain ${ }^{10}$, manifeste, plus encore que les autres "pronoms personnels », une extrême plasticité :

- dans le langage oral ordinaire, il doit être considéré comme le morphème standard de la valeur "locuteur amplifié » (plus courant que le nous canonique des paradigmes de conjugaison qui en apparaît, synchroniquement, comme une variante hypercorrecte) ;

${ }^{7}$ Voir notamment : S. Kuno, E. Kaburaki, « Empathy and Syntax », Linguistic Inquiry, 8-4, 1977.

${ }^{8}$ R. Forest, Empatbie et linguistique, Paris, P.U.F., 1999.

${ }^{9}$ La désignation de personne amplifiée est courante chez les grammairiens depuis l'article d'E. Benveniste «Structure des relations de personne dans le verbe (Problèmes de linguistique générale, I, Paris, Gallimard, 1966).

${ }^{10}$ Le morphème on peut certes référer à un chien ou à une souris (par exemple, l'énoncé T'as vu ça ? On a faim ! de l'arborescence II.a. 2.1.1.), mais c'est bien, semble-t-il, en conférant à son référent, en quelque manière, un caractère anthropomorphique. 
- on peut également, dans des usages souvent présentés comme "rhétoriques» par les grammairiens, prendre toutes les valeurs déictiques des autres «pronoms personnels » (correspondant, dans notre terminologie, tout comme la valeur « locuteur amplifié », à des valeurs d'indexicalité extratextuelle) ;

- il est candidat à un grand nombre de valeurs anaphoriques (correspondant à nos valeurs d'indexicalité intratextuelle);

- il apparaît enfin comme une actualisation privilégiée de la valeur " nominale» - non déictique et non anaphorique - d'un clitique verbal portant le trait [+humain] (dans notre terminologie, la valeur symbolique).

Afin de décrire ces valeurs de on, nous reprendrons les grandes lignes d'une grammaire sémiotique du système des morphèmes clitiques français dits assez improprement « pronoms personnels » esquissée dans un précédent article ${ }^{11}$.

\section{Grammaire sémiotique de on}

Notre grammaire distingue, sur une base strictement différentielle, 103 valeurs du morphème on. Pour en faciliter la lecture, ses arborescences binaires seront fractionnées en 6 sections, désignées par des chiffres romains :

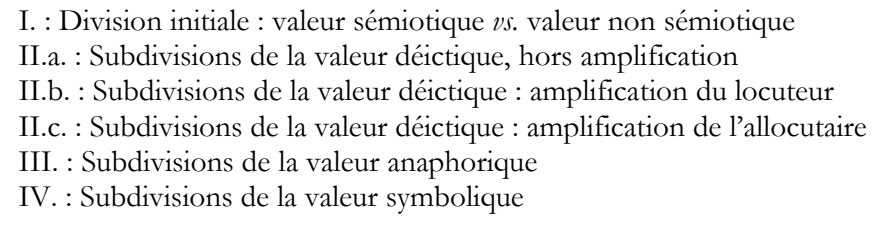

De ce fait, à partir de la deuxième arborescence, la notation numérique des valeurs sera abrégée. Leur numérotation complète - reflétant leur caractère différentiel au sein de l'ensemble de la grammaire - peut être rétablie dans les subdivisions II.a. à IV., en ajoutant, à gauche de la numérotation des valeurs, la numérotation de la branche terminale inachevée de l'arborescence précédente (qui figure en italiques grasses) dominant ces subdivisions.

Les numérotations et les compositions de traits descriptifs figurant en caractères romains gras sont celles actualisées par un morphème. Le niveau de détail auquel parviennent ces arborescences se justifie de l'existence, pour chaque composition terminale de traits, d'énoncés homonymes possibles dont ces traits permettent de différencier le sens - y compris quant aux éléments de sens dont répondent les accords en genre et en nombre au sein du groupe verbal.

Pour des raisons de place, nous n'adopterons pas la schématisation en arbre.

\subsection{Division initiale : valeurs sémiotiques vs valeur non sémiotique (arborescence I.)}

Notre division initiale est celle des traits [+IDX ] (valeur indexicale) et [-IDX] noté encore [+SYM] (valeur non indexicale, ou symbolique).

\footnotetext{
$11 \mathrm{~S}$. Bouquet, «Sémiotique grammaticale et sémantique des (genres de) jeux de langage : les 'pronoms personnels' clitiques en français ", Les genres de la parole, Langages, $\mathrm{N}^{\circ}$ 153, mars 2004.
} 
Simon Bouquet

Le trait [+IDX] se subdivise en deux traits :

- le trait d'indexicalité extratextuelle $([+\mathrm{EXT}])$, subsumant les valeurs dites classiquement de déixis ;

- le trait d'indexicalité intratextuelle ([-EXT]) subsumant les valeurs dites classiquement d'anaphore ${ }^{12}$.

On réservera un traitement particulier à une valeur de on homologue, selon nous, de celle du clitique sujet il dans son emploi dit impersonnel - autrement dit une valeur sémiotiquement vide. En effet, tout comme le clitique il dans il faut, il se peut que, ou il pleut, le clitique on de l'expression on dirait et ses flexions - dans ses emplois synonymes de l'expression il semble et ses flexions - peut être considéré, selon nous, comme «vide», c'est-à-dire comme ayant pour seule fonction sémantique d'occuper une place syntaxique au sein d'une locution figée.

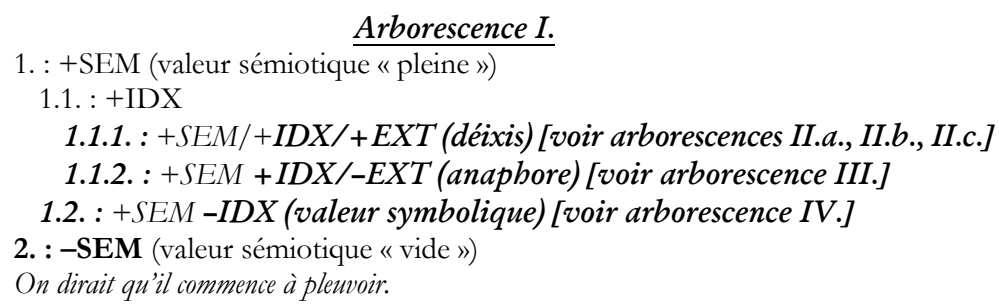

Arborescence I.

1. : +SEM (valeur sémiotique «pleine »)

\subsection{Subdivisions de la valeur déictique $[+I D X /+E X T]$ hors amplification (arborescence II.a.)}

La déixis ou indexicalité extratextuelle peut, d'une manière générale, être interlocutoire, spatiale, temporelle ou ostensive ${ }^{13}$. Seules sont pertinentes pour la grammaire du morphème on - tout comme pour la grammaire des «pronoms personnels » - les valeurs interlocutoires (notées ici [+ITL]) et ostensives (notées ici [-ITL]).

La valeur interlocutoire $[+ \text { ITL }]^{14}$ est la valeur standard de ce que les grammaires nomment ordinairement personnes $d u$ dialogue: cette valeur est subdivisée par les traits suivants :

- $[+\mathrm{LOC} /-\mathrm{EMP}(\mathrm{AMP})]^{15}$ (locuteur non amplifié), sous lesquels se range la valeur standard de $j e^{16}$;

- [+LOC/+EMP(AMP)] (locuteur amplifié), sous lesquels se range la valeur standard de nous;

\footnotetext{
12 On entend anaphore dans son sens étendu, incluant la cataphore.

13 On entend par ostensive une valeur déictique non interlocutoire, non spatiale et non temporelle.

${ }^{14}$ Le trait [+ITL] implique de facto le trait [+HUM] (humain) ; celui-ci n'est toutefois pas requis pour la présente description différentielle.

${ }^{15}$ Les traits [-EMP(AMP)] et [+EMP(AMP)], remplaçant les traits [-AMP] et [+AMP] de notre précédente grammaire, notent que ce que l'on appelle traditionnellement amplification est considéré comme un cas particulier d'empathie. Nous conservons néanmoins la spécification $A M P$ par souci de lisibilité.

16 On donne ici les formes nominatives des morphèmes; ces traits s'appliquent également à leurs formes accusatives ou datives.
} 
- [-LOC/-EMP(AMP)] (allocutaire non amplifié), sous lesquels se range la valeur standard de $t u$;

$-[-\mathrm{LOC} /+\mathrm{EMP}(\mathrm{AMP})]$ (allocutaire amplifié), sous lesquels se range la valeur standard de vous.

Comme on l'a signalé, on peut prendre toutes ces valeurs ${ }^{17}$.

Les valeurs ostensives, dominées par le trait [-ITL] et subdivisées par des traits de nombre et de genre grammaticaux, décrivent le sens déictique que peuvent prendre les morphèmes il, ils, elle, et elles, et semblablement le morphème on.

\section{Arborescence II.a.}

(subdivisant la valeur 1.1.1. de l'arborescence I. [+SEM/+IDX/+EXT])

1. : +ITL (déixis interlocutoire)

1.1.: +ITL/+LOC (déixis interlocutoire du locuteur)

1.1.1.: +ITL/+LOC/-EMP(AMP) (locuteur non amplifié)

1.1.1.1. : +ITL/+LOC/-EMP(AMP)/+MASC

(Pierre se justifie, l'air gêné, devant Paul qui semble étonné de le voir manger goulûment :) On est affamé ! [on $=\mathrm{je}]$

1.1.1.2. : +ITL/+LOC/-EMP(AMP)/-MASC

(Marie se justifie, l'air gênée, devant Paul qui semble étonné de la voir manger goulûment :) On est affamée! [on $=$ je]

1.1.2. : $+I T L /+L O C /+E M P(A M P)$ (locuteur amplifié) [voir arborescence IIb.]

1.2.: +ITL/-LOC (déixis interlocutoire de l'allocutaire)

1.2.1.: +ITL/-LOC/-EMP(AMP) (allocutaire non amplifié)

1.2.1.1. : +ITL/+LOC/-EMP(AMP)/+MASC

(Paul se moque gentiment de Pierre qui mange goulûment :) Eh bé! On est affamé ! $[\mathrm{on}=\mathrm{tu}]$

1.2.1.2. : +ITL/+LOC/-EMP(AMP)/-MASC

(Paul se moque gentiment de Marie qui mange goulûment:) Eh bé! On est affamée! $[\mathrm{on}=\mathrm{tu}]$

1.2.2. : $+I T L /-L O C /+E M P(A M P)$ (allocutaire amplifié) [voir arborescence IIc.]

2. :-ITL (déixis non interlocutoire)

2.1.: -ITL/+SING (non-interlocuteur singulier)

2.1.1. :-ITL/+SING/-GE.DET (genre indéterminé)

(Pierre dit à Paul, en lui montrant un bébé qui tète goulûment son biberon :) T'as vu ça? On a faim! [on = il (neutre)]

2.1.2. :-ITL/+SING/+GE.DET (genre déterminé)

2.1.2.1. : -ITL/+SING/+GE.DET/+MASC

(Pierre dit à Paul, en lui montrant Jean qui mange goulûment :) T'as vu ça ? On est affamé ! [on = il]

2.1.2.2. :-ITL/+SING/+GE.DET /-MASC

(Pierre dit à Paul, en lui montrant Marie qui mange goulûment :) T'as vu ça ? On a est affamée ! [on = elle]

2.2.: -ITL/-SING (non-interlocuteur pluriel)

2.2.1. : -ITL/-SING/-GE.DET

(Pierre dit à Paul, en lui montrant Jean et Marie qui mangent goulûment :) T'as vu ça? On est affamés! [on = ils (neutre)]

2.2.2. : -ITL/-SING/+GE.DET

${ }^{17}$ Ces valeurs de on - hormis la valeur [+LOC/+EMP(AMP)] (= nous) - sont communément considérées comme « rhétoriques » par les grammairiens. 


\title{
2.2.2.1. : -ITL/-SING/+GE.DET /+MASC
}

(Pierre dit à Paul, en lui montrant Jean et Jacques qui mangent goulûment :)

T'as vu ça? On est affamés! [on = ils]

2.2.2.2. : -ITL/-SING/+GE.DET /-MASC

(Pierre dit à Paul, en lui montrant Marie et Anne qui mangent goulûment :)

T'as vu ça? On est affamées! [on = elles]

\subsection{Subdivisions de la valeur déictique : amplification du locuteur (arborescence II.b.)}

L'amplification recouvre, dans son mécanisme sémiotique, de multiples organisations de signifié possibles, qui se laissent décrire comme des constructions de sphères d'empathie. Ainsi, un morphème comme nous ou son «synonyme » on, dont le centre d'empathie est noté dans notre arborescence par la valeur [+LOC] (locuteur : 'moi'), pourra, dans son amplification, équivaloir à 'moi + toi', 'moi + lui', 'moi + vous', 'moi + eux', etc. On décrira ici un palier de régularité de ces constructions : celui qui apparait dans la composition de valeurs standard des «pronoms personnels » et qu'attestent les accords en genre et en nombre au sein du groupe verbal.

Les non-interlocuteurs impliqués dans l'amplification peuvent relever d'une déixis ou d'une anaphore. Aussi, comme on le verra, le processus de l'amplification, lorsqu'il inclut des non-interlocuteurs, se montre-t-il susceptible de composer soit plusieurs types de valeurs déictiques (interlocutoires et non interlocutoires), soit des valeurs déictiques et des valeurs anaphoriques ${ }^{18}$.

\section{Arborescence II.b. \\ (subdivisant la valeur 1.1.2. de II.a. [+SEM/+IDX/+EXT/+ITL/+LOC/+EMP(AMP)])}

\author{
1. : +ALLO (amplification du locuteur à l'allocutaire) \\ 1.1. : +ALLO/+SING (allocutaire singulier) \\ 1.1.1. : +ALLO/+SING/+EXCL (exclusivement) ${ }^{19}$ \\ (Pierre à Marie :) Si tu veux, on déjeune ensemble. $[\mathrm{on}=$ nous $=$ moi + toi $]$ \\ 1.1.2.: +ALLO/+SING/-EXCL (non exclusivement)
}

\footnotetext{
18 Cette arborescence illustre le fait que les indexicalités déictique et anaphorique ressortissent bien à un fait sémiotique commun et majeur.

${ }^{19}$ Un module « genre grammatical » peut être inséré, sur la base des subdivisions suivantes :

1. : +GE.DET (genre grammatical déterminé)

1.1. : +MASC (masculin)

1.2. :-MASC (féminin)

2.-GE.DET (genre grammatical indéterminé)

(le trait [-GE.DET] notant indifféremment un genre réellement indéterminé et la valeur «neutre» du pluriel masculin en français), pour traiter les diverses compositions des traits [+LOC] et [+ALLO]. Par exemple, dans la section 1.1.1.

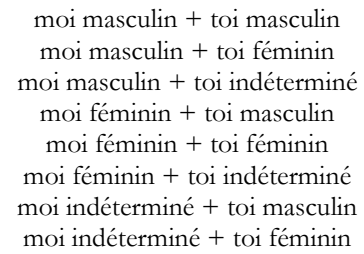


1.1.2.1.: +ALLO/+SING/-EXCL/+TIERSING (incluant un tiers ${ }^{20}$ singulier)

1.1.2.1.1. : +ALLO/+SING/-EXCL/+TIERSING/+DEI ${ }^{21}$ (par déixis) 1.1.2.1.1.1. : +ALLO/+SING/-EXCL/+TIERSING/+DEI/-GE.DET

(Pierre à Marie, voyant une silhouette se diriger vers eux:) On sera au moins trois à la réunion $[\mathrm{on}=$ nous $=\mathrm{moi}+$ toi + quelqu'un(neutre $)]$

1.1.2.1.1.2. : +ALLO/+SING/-EXCL/+TIERSING/+DEI/+GE.DET 1.1.2.1.1.2.1. : +ALLO/+SING/-EXCL/+TIERSING/ +DEI/ +GE.DET/+MASC

(Pierre à Marie, reconnaissant Paul qui arrive :) On sera au moins trois à la réunion.

$[$ on $=$ nous $=$ moi + toi + lui $]$

1.1.2.1.1.2. : +ALLO/+SING/-EXCL/+TIERSING/+DEI/

+GE.DET/-MASC

(Pierre à Marie, reconnaissant Julie qui arrive :) On sera au moins trois à la réunion.

[on $=$ nous $=$ moi + toi + elle $]$

1.1.2.1.2. : +ALLO/+SING/-EXCL/+TIERSING/-DEI (par anaphore)

1.1.2.1.2.1. : +ALLO/+SING/-EXCL/+TIERSING/-DEI/-GE.DET

(Pierre à Marie :) Ma secrétaire m'a dit que quelqu'un a téléphoné pour annoncer sa venue. On sera au moins trois à la réunion . $[$ on $=$ nous $=$ moi + toi + quelqu'un(neutre)]

1.1.2.1.2.2. : +ALLO/+SING/-EXCL/+TIERSING/-DEI/+GE.DET

1.1.2.1.2.2.1. : +ALLO/+SING/-EXCL/+TIERSING/-DEI/+GE.DET/

+ MASC

(Pierre à Marie :) Paul m'a prévenu qu'il viendrait. On sera au moins trois à la réunion.

[on $=$ nous $=$ moi + toi + lui $]$

1.1.2.1.2.2.2. : +ALLO/+SING/-EXCL/+TIERSING/-DEI/+GE.DET/

-MASC

(Pierre à Marie :) Julie m'a prévenu qu'elle viendrait. On sera au moins trois à la réunion

[on $=$ nous $=$ moi + toi + elle $]$

1.1.2.2.: +ALLO/+SING/-EXCL/-TIERSING (incluant plusieurs tiers 22 )

1.1.2.2.1. : +ALLO/+SING/-EXCL/-TIERSING/+DEI (par déixis)

1.1.2.2.1.1. : +ALLO/+SING/-EXCL/-TIERSING/+DEI/-GE.DET

(Pierre à Marie, voyant deux silhouettes se diriger vers eux:) On sera an moins quatre à la réunion $[\mathrm{on}=$ nous $=\mathrm{moi}+$ toi + eux (neutre) $]$

1.1.2.2.1.2. : +ALLO/+SING/-EXCL/-TIERSING/+DEI/+GE.DET

1.1.2.2.1.2.1. : +ALLO/+SING/-EXCL/-TIERSING/+DEI/ +GE.DET/+MASC

(Pierre à Marie, reconnaissant Paul et Jacques qui arrivent :) On sera an moins quatre à la réunion. $[$ on $=$ nous $=$ moi + toi + eux $($ masculin $)]$

1.1.2.2.1.2.2. : +ALLO/+SING/-EXCL/-TIERSING/+DEI/ +GE.DET/-MASC

(Pierre à Marie, reconnaissant Julie et Anne qui arrivent :) On sera an moins trois à la réunion $[\mathrm{on}=$ nous $=\mathrm{moi}+$ toi + elles $]$

1.1.2.2.2. : +ALLO/+SING/-EXCL/-TIERSING/-DEI (par anaphore)

\footnotetext{
20 Par «tiers », on entend ici : un non-interlocuteur.

${ }^{21}$ Le trait [+DEI] est ici une abréviation de la conjonction des traits $[+\mathrm{IDX} /+\mathrm{EXT}]$ appliqués au trait [+TIERSING].

${ }^{22} \mathrm{Par}$ «tiers », on entend ici : un non-interlocuteur.
} 
Simon Bouquet

1.1.2.2.2.1. : +ALLO/+SING/-EXCL/-TIERSING/-DEI /-GE.DET

(Pierre à Marie :) Ma secrétaire m'a dit que deux personnes ont téléphoné pour annoncer leur venue. On sera au moins quatre à la réunion $[$ [on $=$ nous $=$ moi + toi + eux (neutre)]

1.1.2.2.2.2. : +ALLO/+SING/-EXCL/-TIERSING/-DEI /+GE.DET

1.1.2.2.2.2.1. : +ALLO/+SING/-EXCL/-TIERSING/-DEI

/+GE.DET/+MASC

(Pierre à Marie :) Paul et Jacques m'ont prévenu qu'ils viendraient. On sera au moins quatre à la réunion $[\mathrm{on}=$ nous $=\mathrm{moi}+\mathrm{toi}+\mathrm{eux}]$

1.1.2.2.2.2.2. : +ALLO/+SING/-EXCL/-TIERSING/-DEI

/+GE.DET/-MASC

(Pierre à Marie :) Julie et Anne m’ont prévenu qu'elles viendraient. On sera au moins quatre à la réunion $[$ on $=$ nous $=$ moi + toi + elles $]$

1.2. : + ALLO/-SING (amplification du locuteur à l'allocutaire pluriel)

Cette section reprend l'intégralité de la section 1.1. de la présente arborescence, la valeur [+ALLO/-SING] remplaçant ici la valeur [+ALLO/+SING].

$[$ on $=$ nous $=$ moi + vous $(+$ lui/elle/eux/elles $)]$

2. :-ALLO (amplification du locuteur à un non-allocutaire)

Cette section reprend l'intégralité de la section 1.1.2. de la présente arborescence, la valeur [-ALLO] remplaçant ici la valeur [+ALLO/+SING/-EXCL].

[on $=$ nous $=$ moi + lui $/$ elle $/$ eux $/$ elles $]$

On pourrait encore complexifier cette arborescence en subdivisant la valeur [-TIERSING] dans les valeurs [+IDX.HOMO] (indexicalité homogène, subdivisée en [+DEI] ou [- DEI] - valeurs traitées dans la présente arborescence) et [-IDX.HOMO] (indexicalité hétérogène, composant anaphore et déixis - non traitée dans cette arborescence), pour rendre compte de valeurs comme celle de l'énoncé suivant:

(Pierre à Marie, désignant Paul qui arrive :) Avec Jacques qui m’a prévenu qu’il viendrait, on sera au moins quatre à la réunion.

\subsection{Subdivisions de la valeur déictique : amplification de l'allocutaire (arborescence II.c.)}

Les divisions de l'arborescence II.b. permettent également de rendre compte de l'amplification de l'allocutaire (actualisée par vous et par son synonyme on - valeur «rhétorique »). Pour cela, on subdivisera les cinq traits différentiels de l'amplification de l'allocutaire ([+IDX/+EXT/+ITL/-LOC/+EMP(AMP)]) par les mêmes traits que ceux proposés pour les subdivisions de l'amplification du locuteur $([+\mathrm{IDX} /+\mathrm{EXT} /$ +ITL/+LOC/+EMP(AMP)]).

\section{Arborescence II.c. \\ (subdivisant la valeur 1.2.2. de II.a. [+SEM/+IDX/+EXT/+ITL/- LOC/+EMP(AMP)])}

Cette arborescence reprend l'intégralité de l'arborescence II.b. En l'occurrence, le remplacement de la valeur [+LOC] par la valeur [-LOC] aura pour effet de modifier comme suit les valeurs [+ALLO] et [-ALLO] de l'arborescence II.b. : 
1.: + ALLO : amplification de l'allocutaire à un autre allocutaire

1.1. : +ALLO/+SING : amplification de l'allocutaire à un autre allocutaire singulier

1.2. : + ALLO/-SING : amplification de l'allocutaire à un autre allocutaire pluriel

2. :-ALLO : amplification de l'allocutaire à un non-allocutaire (singulier ou pluriel)

\subsection{Subdivisions de la valeur anaphorique $[+I D X /-E X T]$ (arborescence III.)}

Les traits [+IDX/-EXT] (indexicalité intratextuelle) dénotent les valeurs anaphoriques de on (pouvant s'appliquer également à $i l$, ils, elle, elles) ${ }^{23}$.

Qu'elle relève d'une indexicalité exclusivement anaphorique, ou que cette dernière s'y compose avec les valeurs déictiques d'une amplification, l'anaphore peut prendre deux formes :

(1) une anaphore stricte - notée [+IDX/-EXT/-EMP] -, rebaptisée dans notre terminologie anaphore non empathique (l'anaphorisé étant un morphème ou un groupe de morphèmes présent dans le co-texte de l'énoncé); le critère différentiel de cette anaphore non empathique est la commutation possible de on avec des pronoms dits "personnels » (il, elle, ils, elles) et sa non-commutation avec des "pronoms indéfinis » de quantification universelle (tous, chacun, tout un chacun, etc..) ou de quantification existentielle (quelqu'un, certains, d'aucuns, etc.) ;

(2) une anaphore correspondant aux catégories dites classiquement « anaphore associative » ou « anaphore résomptive » - notée [+IDX/-EXT/+EMP] -, rebaptisée dans notre terminologie anaphore empathique ('’anaphorisé n'étant pas strictement un morphème ou un groupe de morphèmes présent dans le co-texte de l'énoncé, mais relevant d'une construction de sphère d'empathie); le critère différentiel de cette anaphore empathique est la non-commutation de on avec des pronoms dits «personnels » et sa commutation possible des «pronoms indéfinis » de quantification universelle ou existentielle ${ }^{24}$.

A la différence de l'anaphore stricte, l'anaphore empathique n'est pas le fait d'un pronom anaphorisant (catégorie $[+\mathrm{PRO} /+\mathrm{GN}]$ de la grammaire générative) mais d'un groupe nominal (catégorie $[-\mathrm{PRO} /+\mathrm{GN}]$ de la grammaire générative). En d'autres termes, cette anaphore se fonde sur une valeur «nominale» de on. C'est pourquoi sa description, dans la section 2. (anaphore empathique) de l'arborescence III., emprunte les traits de la valeur symbolique de on, décrits dans les sections 1.2. et 2. de l'arborescence IV.

\footnotetext{
${ }^{23}$ On n'envisage, regardant on, que des valeurs d'anaphore libre ([+PRO/-ANA] selon la notation de la grammaire générative), on n'étant pas concerné par des valeurs d'anaphore liée ([-PRO/+ANA ]), sinon dans sa forme accusative se, qu'on n'étudie pas ici.

${ }^{24}$ Ainsi, dans les trois énoncés suivants: (1) Dans ce restaurant, on ne fume pas, (2) Dans ce restaurant, on vous sert rapidement, et (3) Dans ce restaurant, on est servi rapidement, le morphème on est respectivement l'anaphore empathique de : «tous ceux qui se trouvent là (énoncé 1), «le personnel de service » (énoncé 2), et « les clients » (énoncé 3).
} 


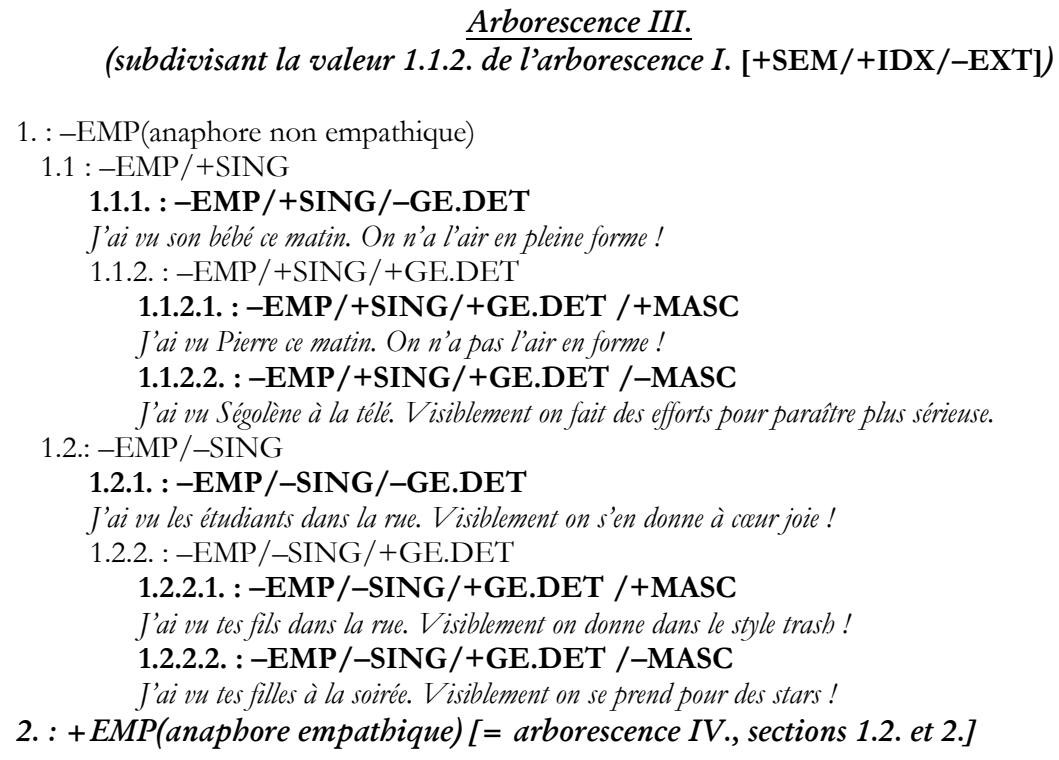

\subsection{Subdivisions de la valeur « symbolique $[-I D X]=[+S Y M]$ (arborescence $I V$.)}

Le trait $[-\mathrm{IDX}]$ note une valeur non déictique et non anaphorique a priori. Cette valeur est dite ici symbolique, selon la terminologie de Peirce - et par commodité on remplacera le trait $[-\mathrm{IDX}]$ par son synonyme : $[+\mathrm{SYM}]$. A ce trait correspond une valeur syntaxique nominale $([-\mathrm{PRO} /+\mathrm{GN}]$ dans la terminologie de la grammaire générative). Elle se laisse subdiviser en :

(1) Une valeur universelle $([+\mathrm{UNI}])$, correspondant à une quantification universelle, se subdivisant en :

- une valeur non empathique ou d'universalité stricte (on $=$ quel que soit $\mathrm{x}$ appartenant à la classe [+HUM]), notée [+UNI/-EMP] ;

- une valeur empathique ou d'universalité restreinte - construite par une sphère d'empathie à l'intérieur de la classe [+HUM] (on = quel que soit x appartenant à la sous-classe empathique $\mathrm{X}$ ) -, notée [+UNI/+EMP].

Dans ces deux versions de la valeur d'universalité, on peut être remplacé, peu ou prou, par des pronoms ou locutions comme tous, chacun, tout un chacun, n'importe qui, personne (dans un énoncé négatif), etc., s'entendant strictement ou dans une sphère restreinte.

(2) Une valeur existentielle (correspondant à la quantification existentielle : on $=$ il existe un $\mathrm{x}$ tel que...) ; dans cette valeur on peut être remplacé par quelqu'un, certains, d'aucuns, etc. Cette valeur, restrictive quant à la classe [+HUM], implique la création d'une sphère d'empathie qui régit cette restriction. Le trait [+EMP] est donc inhérent à cette valeur - c'est d'ailleurs pour cette raison qu'il ne figure pas dans ses subdivisions, n'y jouant pas de rôle différentiel (au même titre que le trait [+HUM] dans l'ensemble de nos notations). 
Les valeurs d'universalité restreinte (valeur 1.2. de l'arborescence IV.) et existentielle (valeur 2. de l'arborescence IV.), tout en étant «nominales» - et parce qu'elles sont nominales - sont les valeurs supports de la valeur anaphorique empathique : c'est pourquoi elles apparaissent dans la section 2. de l'arborescence III (et, de fait, les exemples donnés pour ces valeurs dans l'arborescence IV. sont des exemples d'anaphores nominales).

\section{Arborescence IV.}

(subdivisant la valeur 1.2. de l'arborescence I. [+SEM/-IDX]=[+SEM/+SYM]):

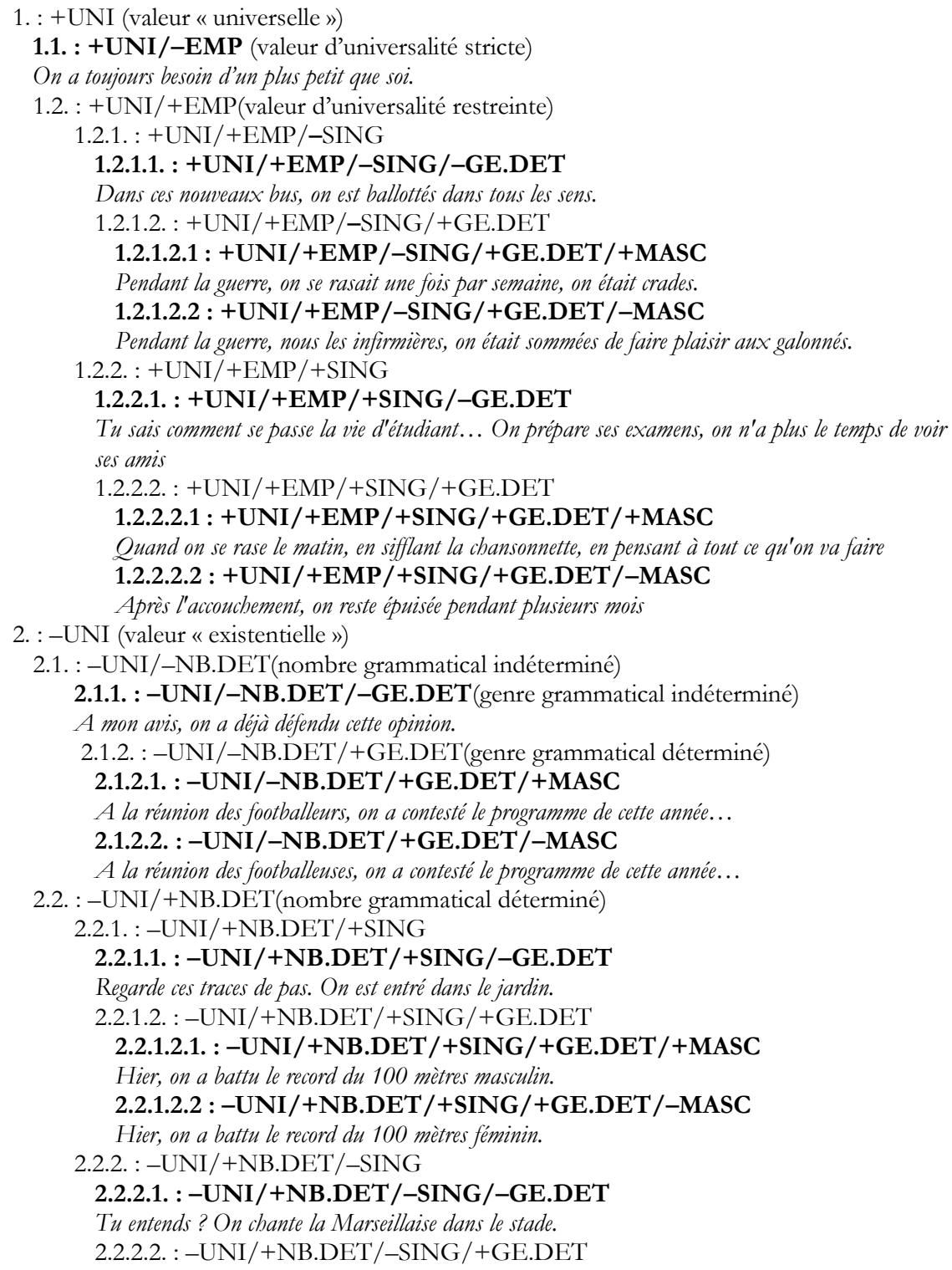


Simon Bouquet

2.2.2.2.1. : -UNI/+NB.DET/-SING/+GE.DET/-MASC

Si cette photo de moi est publiée, je suis sûr qu'on me demandera en mariage!

2.2.2.2.2. :-UNI/+NB.DET/-SING/+GE.DET/+MASC

Si cette photo de moi est publiée, je suis sûre qu'on me demandera en mariage!

\section{En guise de conclusion}

Cette grammaire n'est qu'une esquisse, réalisée dans le cadre de travaux en cours. Dans la mesure où elle ne saurait suffire à analyser, dans tout le détail requis, les corrélations entre grammaire de langue et grammaire des genres, elle devra être complétée ultérieurement par des grammaires sémiotiques des signifiés verbaux avec lesquels on entre en relation de dépendance structurale : signifié de désinence (temps et mode verbaux) et d'auxiliarisation, signifié « lexical » du radical verbal ${ }^{25}$.

Une telle approche devrait faire apparaître, dans des grands corpus, des régularités dans les corrélations entre, d'une part, des traits de genre et, d'autre part, des configurations de traits de ces grammaires sémiotiques.

25 On peut résumer ainsi ces corrélations :

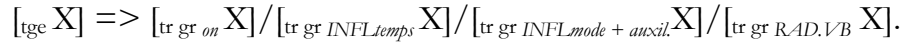

